\title{
Medical Case Reports Journal
}

\section{Successful Management of Dysgeusia by Photobiomodulation (PBM) in a Cancer Patient}

\author{
Juliano Abreu Pacheco ${ }^{* 1}$, Adriana Schapochnik ${ }^{2}$ and Claudia Conforto de $\mathrm{Sa}^{3}$ \\ ${ }^{1}$ Dental Surgeon, Department of Hospital Dentistry, Hospital do Cancer de Ribeirao Preto, Brazil \\ ${ }^{2}$ Physiotherapist, Specialist in Traditional Chinese Medicine, Brazil \\ ${ }^{3}$ Physiotherapist, Brazilian Institute of Intensive Dentistry, Brazil
}

Article info

Received 07 December 2018

Revised 25 February 2019

Published 11 March 2019

*Corresponding author: Juliano Abreu Pacheco, Dental Surgeon, Department of Hospital Dentistry, Hospital do Cancer de Ribeirao Preto, Brazil; E-mail: japacheco@hcancerderibeirao.org. br, coepacheco@gmail.com,

\begin{abstract}
In this case study, the Low Intensity Laser was used in the oral cavity (tongue and salivary glands) and in the radial artery of the wrist for the treatment of dysgeusia. The introduction of Low Intensity Laser Therapy in this case promoted the improvement of dysgeusia that compromised the tasting of food flavors and a significant improvement to the adherence of the therapies proposed by the multidisciplinary team in the control of the disease. This non-invasive and low-cost phototherapy protocol performed a viable alternative in the local stabilization of the oral cavity with significant systemic repercussions.
\end{abstract}

Keywords: Dysgeusia; Oncology; Laser; Cancer; Oral cavity; Taste; Chemotherapy; Photobiomodulation

\section{Introduction}

Dysgeusia is variably defined as an abnormality detrimental to taste sensations that causes unpleasant palate alteration, or a distortion or perversion of the sense of taste. It is closely linked to changes in taste and smell as they provide sensory information correlated with sensory pleasure that are directly affected by cancer-related therapies [1].

These changes in taste and smell in cancer patients are prevalent problems due to therapeutic interventions that affect quality of life, leading to malnutrition, weight loss and, in severe cases, significant morbidity [2].

Dysgeusia is assessed clinically by measuring the detection or recognition threshold values for the five sub-gustations (Figure 1): sweet, bitter, acid, salty and umami (the taste of protein-rich foods), whether by the application of filters (paper taste strips impregnated with various concentrations of basic taste) or, in the laboratory environment, with the use of an instrument called elegance.
In fact, these qualitative changes are more reliable when reported through patient complaints, interviews and clinical observations [3]. This sensory system in cancer patients is orchestrated by numerous exogenous and endogenous factors that interact with one another. Faced with this, these factors are responsible for taste and odor abnormalities due to this interconnection. However, this damage to sensory receptor cells is abnormal, but the etiology of these is related to the neuronal activities causing the abnormalities of taste and odor in these patients.

And according to scientific research, these disorders are probably multifactorial, although it is conclusive by many authors that chemotherapy and radiotherapy can cause disruption to taste in the destruction of olfactory and taste cell receptors. And this cellular damage may manifest in three ways: (a) a decrease in the number of normal recipient cells, (b) structural cell alteration or changes in the receptor surface, and (c) disruption in neural coding. 


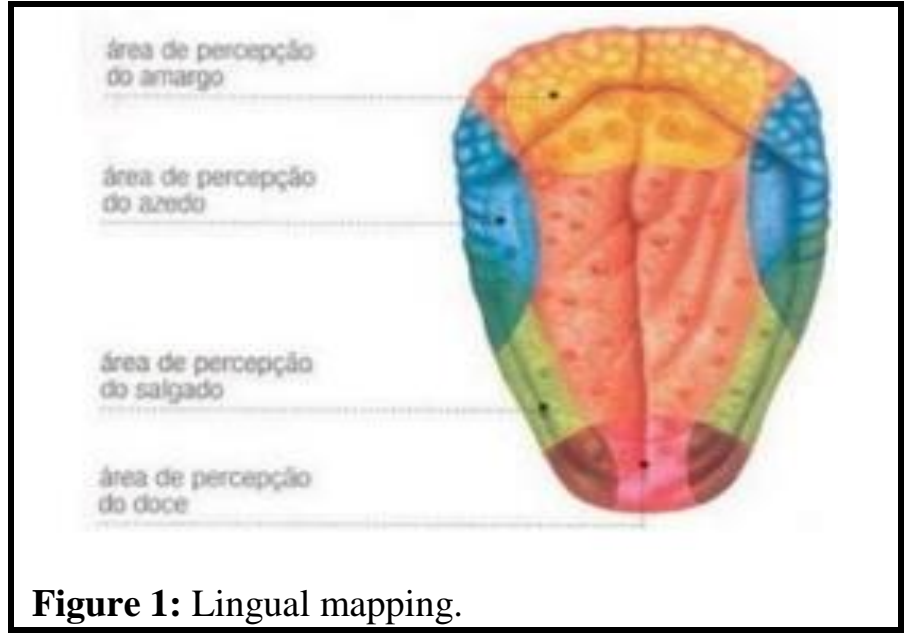

Photobiomodulation (PBM) is a non-invasive infrared and red-light therapy that is absorbed by endogenous chromophores, triggering biological reactions that are not thermal or cytotoxic by means of photochemical or photophysical events, leading to physiological changes. Radiation emitted at certain wavelengths can modulate cells and bring physiological balance to tissues [4].

Photobiomodulation is known as low intensity laser therapy because the light used is low power compared to other forms of laser health treatments, which are used in other areas of medicine, such as surgery [4]. In recent studies it has been reported that the mechanism of action of PBM is closely related to an action on cytochrome c oxidase $(\mathrm{CcO})$ in the mitochondrial respiratory chain, facilitating the electron transport that promotes an increased transmembrane proton gradient, stimulating the production of triphosphate of adenosine (ATP), increasing the functions of cellular metabolism $[5,6]$.

Low intensity laser therapy improves tissue regeneration by acting at different stages of lesion resolution, including the inflammation and re-modeling phases [7]. Some beneficial effects such as immunosuppression-immunostimulation, autoimmune disease and nerve regeneration have been reported in the literature. It is gaining recognition as a viable treatment option for a diverse range of diseases and conditions characterized by injury, degeneration, inflammation and pain [8]. "The laser was not able to replace many of today's techniques and physical modalities; however, it can be used together to improve the health of patients [9]." On the other hand, the complementary function, Ilib, triggers an antioxidant system composed by enzymes. The main metallo enzyme superoxide dismutase, or SOD $\mathrm{ZnCu}$, is the largest antioxidant agent [10] that we have and fifth volume enzyme in the human body. However, according to a recent review, evidence suggests that the enzymes catalaseperoxidase and ceruloplasmin also absorb the red laser which potentiates other enzymes, which obviously further potentiates the antioxidant properties of these enzymes when irradiated in the ILIB process.

\section{Case Report}

A 63-year-old white female RQS diagnosed with multifocal invasive right breast carcinoma in May 2017. Tumorectomy and lymphadenectomy of axillary lymph nodes were performed in June 2017, followed by treatment with chemotherapy (QT) 4 cycles (docetaxel), ending in January 2018. Patient used oral dexamethasone, with clinical status of G1 mucositis, hyporexia, G2 diarrhoea, G1 myalgia, skin lesions and tearing. In October of 2017, during Q3 C3, he presented to the Dentistry Service of the Hospital do Cancer for diagnosis and follow-up of reflexes from oncological therapies. In the initial consultation, the use of a provisional superior prothesis (5 implants) and lower teeth under normal conditions was observed, but the patient's main complaint was the loss of the palate function, called dysgeusia (Figure 2), which made the diet difficult habitual. It reported that the sensory function of the tongue had been compromised which affected the perceptions of salt and sweet flavors. Upon confirmation of the sensorial alteration of the tongue, initial topical therapy with the use of $3 \mathrm{X}$ mouthwashes per day of chamomile tea at room temperature [11] was proposed; advice on diet in order to avoid acidic and peptic foods; photobiomodulation (low intensity laser therapy) in the oral cavity; and photoenteral via ILIB Intravascular Irradiation off blood [12].

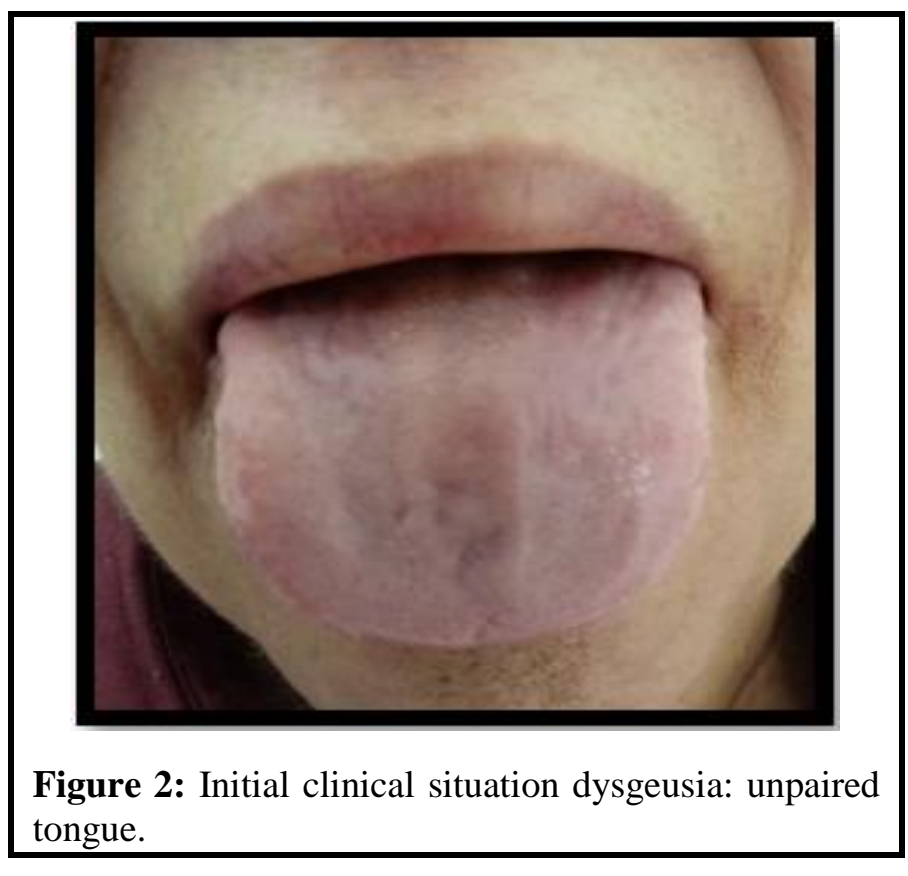


Citation: Pacheco JA, Schapochnik A, Conforto de Sa C. Successful Management of Dysgeusia by Photobiomodulation (PBM) in a Cancer Patient. Med Case Rep J 2019; 2: 114. doi: 10.31531/2581$\underline{5563.1000114}$

The main objective of this study was to perform controlled phototherapy in the lingual region and the major salivary glands (parotid, submandibular, sublingual), non-invasive in this patient of the Cancer Hospital of Ribeirão Preto (SP), through the EC laser, DMC brand, useful red laser emitter: $100 \mathrm{~mW} \pm 20 \%$, red laser wavelength $660 \mathrm{~nm} \pm 10 \mathrm{~nm}$, photobiomodulator effect, with specific protocol, and use of the photothermal/Ilib (Intravascular Laser Irradiation of blood) function in the radial artery of the wrist as a complementary action.

\section{Clinical conduct (Protocol')}

- Before each session, the patient's initial blood pressure was evaluated.

- Hygiene of the oral cavity with clodiaxidine $0.12 \%$ by digital friction, using the sterile gauze.

- Perception of taste flavors through manual technique.

- Application of the Low Intensity Laser (LIB) laser XT, DMC brand, useful red emitter laser power: $100 \mathrm{~mW} \pm 20 \%$, red laser wavelength $660 \mathrm{~nm} \pm 10$ $\mathrm{nm}, 1$ joule (10 seconds)-red 10 seconds in bilateral salivary glands (parotid, sublingual and submandibular), and in the lingual region (lateral border 9 points 1 joule/point and 2 joules on the back).

- Complementary 15-minute photoenteral therapy (Ilib) in the radial artery of the wrist.

- Checking the final blood pressure.

- The sensory measurement of the taste buds was materialized by personalized scale of the Hospital of Cancer of Ribeirão Preto, being marked by small doses ( 0.25 grams) of the flavors related to salt, sweet, sour and bitter: Scale proposed in Table 1 (Palate reduced 1; sharp palate; 2; normal taste 3) The patient was followed weekly ( 2 times / week) for 2 months on alternate days for the application of Lib (Figure 3a), and simultaneously with Ilib therapy (Figure 3b) according to the indoctrination proposed by co-author ${ }^{2}$ on "patient management "Who need greater regulatory control of general health status.
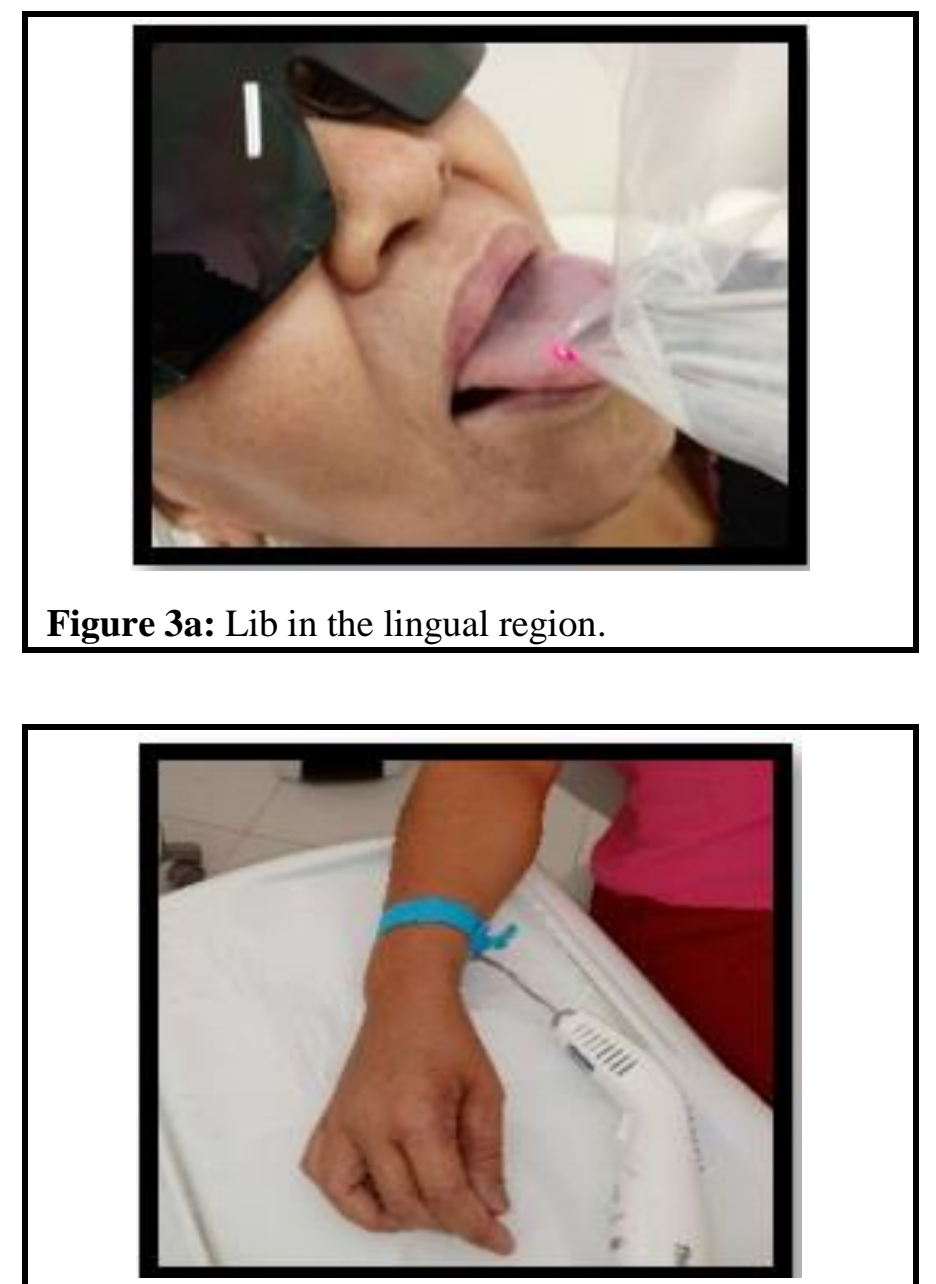

Figure 3b: Ilib in the radial artery of the right wrist.

The results obtained were increasing until reaching the normalization of the sensory function before the proposed period initially, according to Table 1 below:

Table 1: Proposed scale.

\begin{tabular}{|c|c|c|c|}
\hline Chronology-Date & Scale Palate T1 & Protocol $^{\mathbf{1}}$ & Scale Palate T2 \\
\hline I- 05/10/2017 & 1 & LLL/ILIB & 1 \\
\hline II- 10/10/2017 & 1 & LLL/ILIB & 2 \\
\hline III- 17/10/2017 & 2 & LLL/ILIB & 2 \\
\hline IV- $19 / 10 / 2017$ & 2 & LLL/ILIB & 2 \\
\hline V- 24/10/2017 & 2 & LLL/ILIB & 2 \\
\hline VI- 26/10/2017 & 2 & LLL/ILIB & 2 \\
\hline VII- 31/10/2017 & 2 & LLL/ILIB & \\
\hline
\end{tabular}


Citation: Pacheco JA, Schapochnik A, Conforto de Sa C. Successful Management of Dysgeusia by Photobiomodulation (PBM) in a Cancer Patient. Med Case Rep J 2019; 2: 114. doi: 10.31531/2581$\underline{5563.1000114}$

\begin{tabular}{|c|c|c|c|}
\hline VIII- 09/11/2017 & 2 & LLL/ILIB & 2 \\
\hline IX- $14 / 11 / 2017$ & 2 & LLL/ILIB & 2 \\
\hline X-21/11/2017 & 2 & LLL/ILIB & 2 \\
\hline XI- 23/11/2017 & 2 & LLL/ILIB & 2 \\
\hline XII- 28/11/2017 & 2 & LLL/ILIB & 2 \\
\hline XIII- 30/11/2017 & 2 & LLL/ILIB & 3 \\
\hline XIV- 07/12/2017 & 2 & LLL/ILIB & 3 \\
\hline XV- $12 / 12 / 2017$ & 3 & LLL/ILIB & \\
\hline
\end{tabular}

Note: Scale Palate T1: Benchmarking done before phototherapy; Protocol' : Clinical conduct cited above the table; T2 Palate Scale: Measurement performed at the end of the consultation; LLL: Low Level Laser; ILIB:

Intravascular Laser Irradiation of Blood.

\section{Discussion}

In this study, the initial proposal was to promote a noninvasive dosing through local and systemic photobiomodulation aiming at the partial or total reestablishment of the sensory dysfunction of the patient. The results obtained after the first application of the laser (lib and ilib) demonstrated after 3 days the recovery of the palate, previously numbed by oncological therapies. There was an increase in flavors (mainly related to sweet and salty) that positively impacted the enteral quality of life of the same, even relativizing the lack of balance of the taste buds in the extremism of the tastes.

This accentuation produced a better latency of oncoterapic medications, higher results of laboratory markers and a fundamental impact on self-esteem, which allowed a better resumption of quality of life. Chemotherapy has a severity of dysgeusia by altering the structure of the pores of the taste (resulting in the interrupted delivery of flavor molecules to the recipient cells) or causing a thinning of the papilla epithelium [13]. Cancer treatment can also affect neuronal activities as well as receptor cells. In addition, antineoplastic drugs can damage neuronal cells, thereby modifying the afferent pathways that trigger the taste. Recent research has indicated that lipid peroxidation of oral epithelial cells may contribute to the production of carbonyls that cause a metallic taste sensation. Bitter and metallic sensations may also originate from the toxicity of chemotherapeutic drugs.

Many of these drugs have bitter-tasting compounds that can enter the mouth through the plasma crevicular fluid or may diffuse capillaries to the back of the palate. Drugs that are not anti-neoplastic agents, including antibiotics and analgesics (which are often taken by cancer patients to minimize side effects) may also influence taste perception. And other possible causes of unpleasant taste changes that these patients may present, formerly produced by poor oral hygiene, infection, postnasal drip, gastrointestinal reflux and oral mucositis [14] may be highlighted. Currently, the Low Intensity Laser (LBI) is being used for the global recovery of the patient in several specialties of the health area; and their responses are considered beneficial $[15,16]$ in a variety of different modalities, due to their photobiomodulatory effect [17].

Specifically, this mechanism when triggered in the craniofacial region is related to "neural repair and neurogenesis "Not only in the formation of new brain cells, but also in" synaptogenesis", which is the formation of new connections between existing brain cells [18]. Therefore, it should be noted that the systemic and localized conditions during cancer treatment therapy contribute to the reduction of the sensory capacity of the palate, causing transient or definitive dysgeusias, and in this niche of patients who use chemotherapeutic drugs these functions are altered often with a negative influence on the organic and emotional well-being of these people.

When it acts at the cellular level, the low power laser causes biochemical, bioelectric and bioenergetic modifications, influencing the increase of metabolism, cell proliferation and maturation, the amount of granulation tissue and the decrease of the inflammatory mediators, inducing the process of healing [19]. This radiation emitted by the Low Power Lasers has demonstrated analgesic, anti-inflammatory and healing effects and is therefore widely used in the tissue repair process, due to the low energy densities used and wavelengths capable of penetrating the tissues [20,21]. And when the molecule is absorbed by light allows an increase in cellular metabolism, characterized by stimulation of photoreceptors in the mitochondrial 
Citation: Pacheco JA, Schapochnik A, Conforto de Sa C. Successful Management of Dysgeusia by Photobiomodulation (PBM) in a Cancer Patient. Med Case Rep J 2019; 2: 114. doi: 10.31531/2581$\underline{5563.1000114}$

respiratory chain, changes in cellular ATP levels, release of growth factors and collagen synthesis [22,23].

And in this study the patient returned to perform the normal and nutritional tasks with a certain success after the $1^{\text {st }}$ application and the evolution became a growing in the next 13 consultations until arriving at the normalization in the $14^{\text {th }}$ and consequent stabilization of the picture. The dysgeusia called dysgeusia, began as Ageusia (absence of flavor), evolved to Hypogeusia (low perception of the flavors), then to a Hypergeusia (high taste sensitivity), getting to oscillate between the flavors (Dysgeusia) until the normalization of sensory function of the tongue (Figure 4).

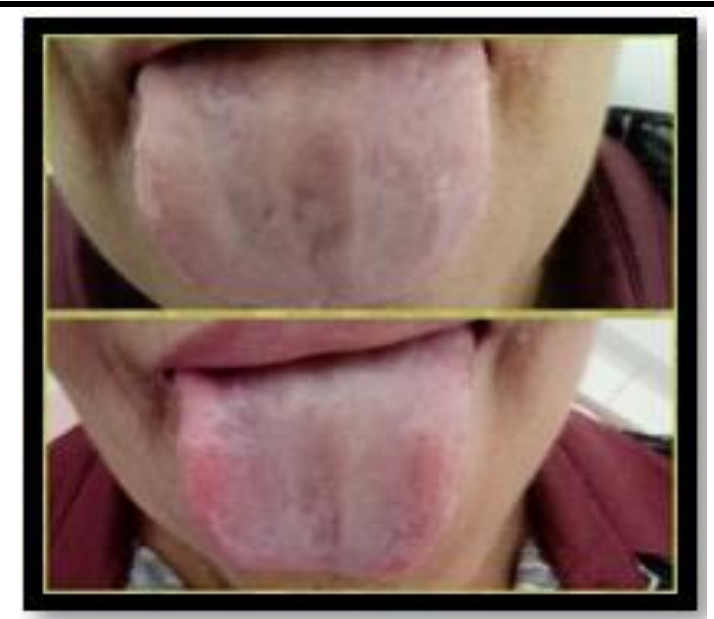

Figure 4: Initial and final stage with return of sensory function.

\section{Conclusion}

The clinical evolution of this specific case is undeniable through photobiomodulation, and it is important the multidisciplinary involvement of all the staff in their various specialties that contributed in an assertive way to stabilize the picture. Although the clinical results look very promising, and the low-power laser fits perfectly into the realm of technological therapy, care must be taken not to regard it as a new panacea. The ideal that this technology continues to perpetuate advances in the oral cavity, from which the systemic repercussions potentiate benefits radiated to other sites fundamental to the maintenance of health.

\section{Conflict of Interest}

None declared.

\section{Funding}

None declared.

\section{References}

1. Heckmann SM. Zinc gluconate in the treatment of dysgeusia: A randomized trial. J Dent Res 2005; 84: 35-38.

2. Halyard M. Taste and odor changes in cancer patients-real problems with few solutions. J Suporte Oncol 2009; 7: 68-69.

3. Hong JH. Abnormalities of taste and odor in cancer patients. J Suporte Oncol 2009; 7: 58-65.

4. Bensadoun RJ, Nair RG. Low Level Laser Therapy in the Management of Mucositis and Dermatitis Induced by Cancer Therapy. Photomed Laser Surg 2015; 33: 487-491.

5. Karu TI. Multiple cytochrome $\mathrm{c}$ oxidase roles in mammalian cells under the action of red and IR-A radiation. IUBMB Life 2010; 62: 607-610.

6. Khakh BS, Burnstock G. The double life of ATP. Sci Sou 2009; 301: 84-92.

7. Hawkins D, Abrahamse H. Biological effects of helium-neon laser irradiation on normal and injured human skin fibroblasts. Photomed Laser Surg 2005; 23: 251-259.

8. T Dai, Tegos GP, Z Lu, et al. Photodynamic therapy for Acinetobacter baumannii infections in mice. Antimicrob Agents Chemother 2009; 53: 39293934.

9. Thiel DC. Low power laser therapy: An introduction and a review of some biological effects. The J CCA 1989; 30.

10. Silvério L Sandra. IBRATE Technology College, Analgesia by Acupuncture 2013.

11. JB Franco, Vaccarezza GF. Importance of dental treatment in the patient of head and neck surgery.

12. Basic Conceptions on Intravenous Laser Therapy with He-Ne (ILIB), JE da Conceição 2014.

13. Silva GB, Sacono NT, Othon-Leite AF, et al. Effect of low intensity laser therapy on the release of inflammatory mediators during oral mucositis induced by chemotherapy: A preliminary randomized study. Lasers Med Sci 2015; 30: 117126.

14. Hong JH. Abnormalities of taste and odor in cancer patients. J Suporte Oncol 2009; 7: 58-65.

15. Sindi SCE, Fokkens J, Ngandu T, et al. The CAIDE dementia risk score app: The development of an evidence-based mobile application to predict dementia. $13^{\text {th }}$ International Geneva/Springfield Symposium on Advances in Alzheimer Therapy; 2014; Geneva, Switzerland.

16. Bellou V, Belbasis L, Tzoulaki I, et al. Systematic evaluation of the associations between environmental risk factors and dementia: An 
Citation: Pacheco JA, Schapochnik A, Conforto de Sa C. Successful Management of Dysgeusia by Photobiomodulation (PBM) in a Cancer Patient. Med Case Rep J 2019; 2: 114. doi: 10.31531/2581$\underline{5563.1000114}$

umbrella review of systematic reviews and metaanalyses. Alzheimers Dement 2016.

17. Kaplan GB, Vasterling JJ, Vedak PC. Brain-derived neurotrophic factor in traumatic brain injury, posttraumatic stress disorder, and their comorbid conditions: role in pathogenesis and treatment. Behav Pharmacol 2010; 21: 427-437.

18. Pacheco JA, Bezinelli LM. The photobiomodulatory therapy and ilib in the repair of encephalic cisterns and progressive cognitive restoration in a patient with traumatic brain injury. Med Case Rep 2018; 4: 73.

19. Silva EM, Gomes SP, Ulbrich LM, et al. Histological evaluation of low-intensity laser therapy in the healing of epithelial, connective and bone tissues: experimental study in rats. Rev SulBras Odontol 2007; 4: 29-35.

20. Barros FC, Antunes SA, Figueredo CMS, et al. Low intensity laser on periodontal healing. $\mathrm{R} \mathrm{Ci} \mathrm{Med}$ Biol 2008; 7: 85-89.

21. Catao MHCV. The benefits of low intensity laser in the dentistry clinic in stomatology. Rev Bras Patol Oral 2004; 3: 214-218.

22. Posten W, Wrone DA, Dover JS, et al. Low-level laser therapy for wound healing: Mechanism and efficacy. Dermatol Surg 2005; 31: 334-340.

23. Kreisler M, Ann B, Christoffers AB, et al. Low level $809-\mathrm{nm}$ diode laser-induced in vitro stimulation of the proliferation of human gingival fibroblasts. Lasers Surg Med 2002; 30: 365-369.

This manuscript was peer-reviewed

Mode of Review: Single-blinded

Academic Editor: Dr. Fadi Farhat

Copyright: (02019 Pacheco JA, et al. This article is distributed under the terms of the Creative Commons Attribution 4.0 International License (http://creativecommons.org/licenses/by/4.0/), which permits unrestricted use, distribution, and reproduction in any medium, provided you give appropriate credit to the original author(s) and the source, provide a link to the Creative Commons license, and indicate if changes were made. 